Research manuscript

\title{
Three dimensional coaxial multi-nozzle device for high-rate microsphere generation
}

\author{
Sravani Jaligama ${ }^{1}$ and Jun Kameoka ${ }^{1, *}$ \\ 1 Electrical and Computer Engineering, Texas A\&M University, College Station, Texas, 77840, USA; \\ jali143@tamu.edu, jaligama.sravani@gmail.com \\ * Correspondence: kameoka@tamu.edu; Tel.: +1-979-845-7564
}

\begin{abstract}
Tissue engineering is an emerging field of research due to its growing impact on the regeneration of tissue injured from damage and organ failure. Small hydrogel microspheres containing regenerative cells and stimulators can be used as building blocks to regenerate tissue by injecting them into damaged areas; a microneedle is used to minimize the incision and any associated infections. To address the need for production of a large number of microspheres for use in tissue engineering, we developed a 3D coaxial multi-nozzle flow-focusing device fabricated via a simple 3D-printing method that demonstrates a high rate of microsphere production per nozzle. This device has six coaxial microscale nozzles that produce hydrogel microspheres. Two individual parts - the inlet and nozzle - can be made separately by a 3D printer and bonded together using uncured photo-curable resin as glue. The dimensions of the microspheres are between $100 \mu \mathrm{m}$ and $1,200 \mu \mathrm{m}$. They are produced by adjusting the flow rate ratio between the dispersed and continuous media. A flow rate ratio of 180 demonstrated the highest microsphere production rate of $2.12 \mathrm{e}+5$ microspheres per sec $(0.25 \mathrm{~mL} / \mathrm{min})$. This microsphere production rate per nozzle is four times higher than that of currently available devices.
\end{abstract}

Keywords: 3D coaxial, multi-nozzle, flow-focusing, high- rate, microsphere, tissue engineering, stereolithography

\section{Introduction}

Tissue and organ failure are major health problems induced by injury or other types of damage. Tissue damage accounts for half of the total cost of health care in the US [1]. The available treatment options include transplantation, surgically implanted artificial prostheses, long-term therapy using drugs, etc. However, complete recovery of damaged tissue is quite difficult, even after treatment over a prolonged period of time. Tissue engineering is an emerging field of research due to its growing impact on the recovery of damaged tissue from injury and organ failure. Microsphere-based scaffolds serving as building blocks for tissue engineering have been investigated extensively in the last two decades, owing to their benefits such as the high level of control they allow over geometry, as well as their reproducibility, dimensions, and mechanical and surface properties [2-4].

Microspheres are droplets with volumes in the order of $0.5 \mathrm{~nL}$ that are commonly used as microreactors to induce small-scale reactions [5-9]; they are also efficient microcarriers of cells and implantable drug carriers [2, 10-11] for tissue engineering applications. These microspheres are commonly made by microfluidic devices via the shear force between two or more immiscible liquids at a T-junction [3] or cross-junction [4, 12-13] to create discrete spheres. Planar microfluidic devices are made of materials like poly(dimethylsiloxane) (PDMS), glass, or poly(methyl methacrylate) (PMMA) [3-4, 12-13] to produce monodisperse spheres. The production rate is determined by flow rates of continuous and meniscus mediums.

Because a great number of microspheres are needed for tissue engineering applications [14-19], a very high microsphere production rate is necessary [20]. A number of devices designed to meet this 
need have been developed. For instance, planar PDMS devices are operated in a high flow-rate regime for sizeable microsphere generation rates using parallel devices, but sphere generation can become unstable and the devices are prone to damage under high pressure [21]. Other devices with large numbers of microsphere generators on a single silicon wafer have achieved relevant microsphere production rates [22-30]. These devices involve complicated structures like ladders or tree-structured channels integrated with microsphere production nozzles. As such, they have an inherent disadvantage in that single-nozzle fouling can disrupt the fluidic pressure distribution, resulting in non-uniform microsphere generation [31]. In our design, this problem is avoided because the flow in one channel is independent from the others due to the individual inlets. Another issue is that the precise alignment of multiple complicated nozzle layers is challenging. This complication degrades the repeatability of channel fabrication, and incomplete bonding significantly reduces the performance of nozzle devices [24]. Fabrication of microsphere production devices made of glass substrate is time-consuming, laborious, and complex, due to difficulties inherent in glass etching. Injection molding, hot embossing, and micro-milling have all been used to make devices from thermoplastics such as polymethylmethacrylate (PMMA) and polycarbonate (PC), but hightemperature fusion bonding between two layers is prone to channel sagging and collapse [32-33].

A possible solution to simplifying fabrication of this type of device could be using a 3D printer to create three-dimensional channels and nozzle microsphere generators capable of sustaining high pressures. A coaxial flow-focusing device design can fabricate smaller-diameter microspheres at a higher production rate [34-36]. To date, coaxial flow-focusing structures have mostly been fabricated with glass capillaries or multiple layers of SU-8 or PDMS; they still require complicated alignment and fabrication processes [39-41]. A single-nozzle 3D-printed device has already been developed and is known to produce monodisperse collagen microspheres with good cell compatibility for applications in tissue engineering [34-35, 42]. However, the production rate achieved using a single coaxial nozzle does not meet the requirements of the tissue engineering field.

In this study, we developed a new 3D-printing fabrication approach to produce a 3D multinozzle device with six coaxial microsphere generators capable of creating a large volume of polyethylene glycol diacrylate (PEGDA) spheres. This multi-nozzle device can stably and reliably produce microspheres with a production rate as high as $2.2 \mathrm{e}+5$ microspheres/sec. Using additive manufacturing technology, we are able to easily and precisely fabricate the microfluidic single-nozzle device with the minimal alignment required.

\section{Materials and Methods}

\subsection{Materials preparation}

The continuous medium contained a light mineral oil (Sigma-Aldrich with a 2\% (v/v) span 80 surfactant (Sigma-Aldrich). The dispersed medium was made of poly(ethylene glycol) diacrylate (PEGDA) (Sigma-Aldrich, molecular weight 700) with $3 \% \quad(\mathrm{v} / \mathrm{v})$ 2,2-dimethoxy-2phenylacetophenone (Sigma-Aldrich) acting as a photo-initiator.

\subsection{Device design and fabrication}

In a coaxial geometry, the shear stress from the continuous media pinches and breaks up the discrete media as it flows through the coaxial orifice into microspheres. In our device, the Reynolds number (Re) and Weber number (We) of continuous the media were 150 and 100, respectively. Hence, the flow was still in the laminar flow regime with the dynamics dominated by the inertial over the viscous effects. There were two dominant forces at the orifice: (1) the inertial stress exerted by the growing microspheres on the continuous flow downstream; and (2) the stress from the continuous flow pinching the dispersed flow, determining the conditions for the breakup. At the nozzle orifice, the balance between these two forces determined the condition for the final diameter of the microspheres as [41]: 


$$
\frac{\mathrm{d}_{\mathrm{b}}}{\mathrm{D}} \alpha\left(\frac{\mathrm{Q}_{\text {inner }}}{\mathrm{Q}_{\text {outer }}}\right)^{2 / 5}
$$

where $d_{b}=$ the diameter of the microsphere, $D=$ the orifice diameter, and $Q=$ the flow rates for the inner and out outer nozzle liquids.

Figure 1a shows the optical images of the actual device with six nozzles. Figure $1 \mathrm{~b}$ includes a schematic of the magnified nozzle portion of the device. The device consists of six sets of nozzle structures for continuous (outer) and dispersed (inner) media. A high-resolution 3D printer from ENVISIONTEC, INC was used to fabricate two separate multi-nozzle device parts with an HTM140M resin material. These two separate parts (see Figures 1c and d) were then easily bonded together by locking the alignment notches on each part (see Figure 1e). Since the laminar flow condition determined by the "Re" value was required in the vicinity of the orifice region for stable and monodisperse microsphere generation, the inner channel widths leading to the orifice were designed to be $250 \mu \mathrm{m}$.
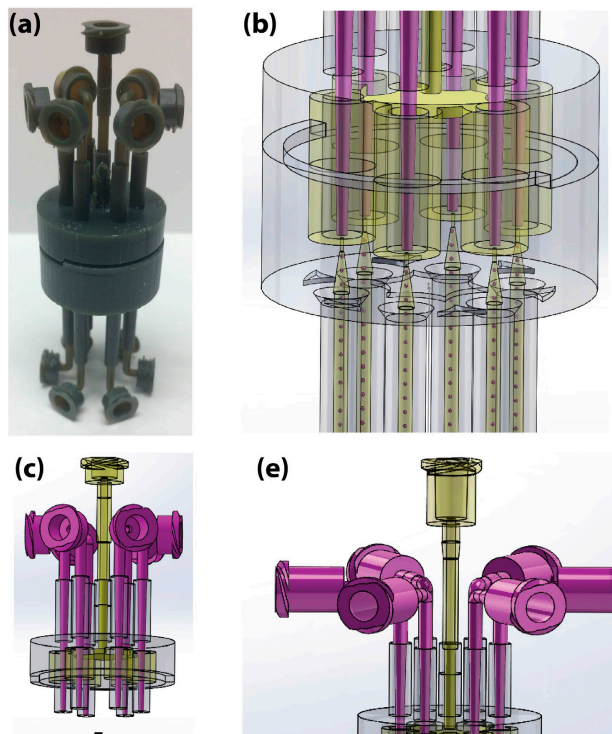

(e)

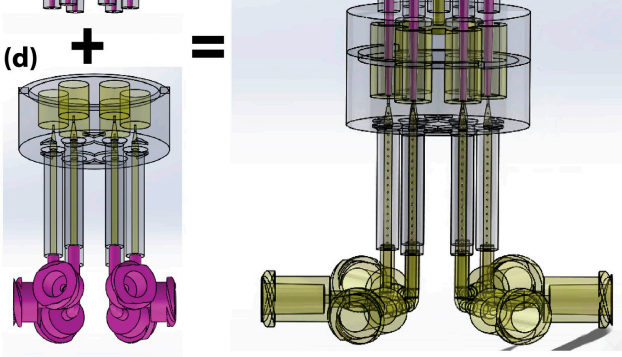

Continuous media (oil)

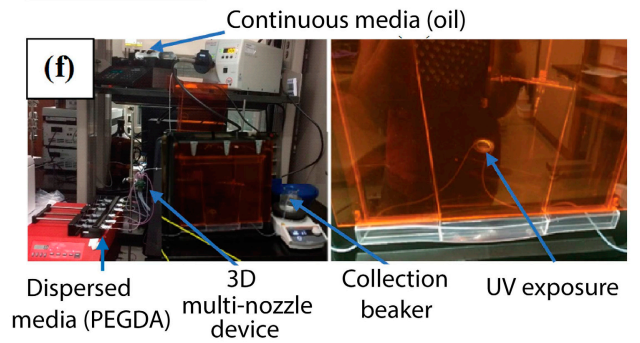

Figure 1. Photo image and schematic of the multi-nozzle device. (a) Photo image of the six coaxial nozzle device; (b) Schematic diagram of the coaxial flow-focusing parts; (c and d) Schematic diagrams of two separate parts made by the 3D printer; (e) Schematic diagram of two parts bonded together based on the alignment notches to form the six-nozzle device; and (f) Experimental setup highlighting various components such as syringe pumps for continuous and dispersed media, the multi-nozzle 
111

device, an outlet tubing inside a UV box that blocked the UV radiation from escaping, and the collection beaker for the spheres $\square$ = Mineral oil $\square=$ PEGDA solution.

The inner diameter of the channels in the cylindrical part that pumps continuous media was set to $750 \mu \mathrm{m}$ to maintain laminar flow, and was still large enough that small-diameter variations did not significantly affect the pressure distribution. The diameter of the microsphere produced followed Eq. 1 and was proportional to the diameter of the orifice and ratio of the flow rate of the inner to outer liquid. For tissue engineering applications, only microspheres with a diameter smaller than $150 \mu \mathrm{m}$ can be injected into tissue using conventional 18, 20, and 24-gauge needles with minimal shear stress on the spheres [43]. Therefore, a smaller orifice diameter is desired to produce smaller microspheres. Due to the resolution limit of the 3D printer, the minimum orifice dimension was $250 \mu \mathrm{m}$, which could produce microspheres below $150 \mu \mathrm{m}$ in diameter, based on Eq.1. Male Luer lock connectors were used to connect the inlets and outlets of the device to the syringe pump, preventing leaking at high flow rates.

The bright field images of the six individual orifices made by the 3D printer are shown in Figure $2 \mathrm{a}$ to $\mathrm{f}$ and the corresponding diameters are listed in Table. 1 . The diameters of the nozzles provide us with information about the variation and repeatability of the device's fabrication. The average diameter of the orifice (D) was $229 \pm 14 \mu \mathrm{m}$. Since the theoretical XY-axis resolution of the 3D printer was $30 \mu \mathrm{m}$, any variations in the dimensions of each nozzle from the average values were within the standard error of the 3D printer. These variations in orifice diameters were acceptable and did not significantly affect the diameters of the microspheres.
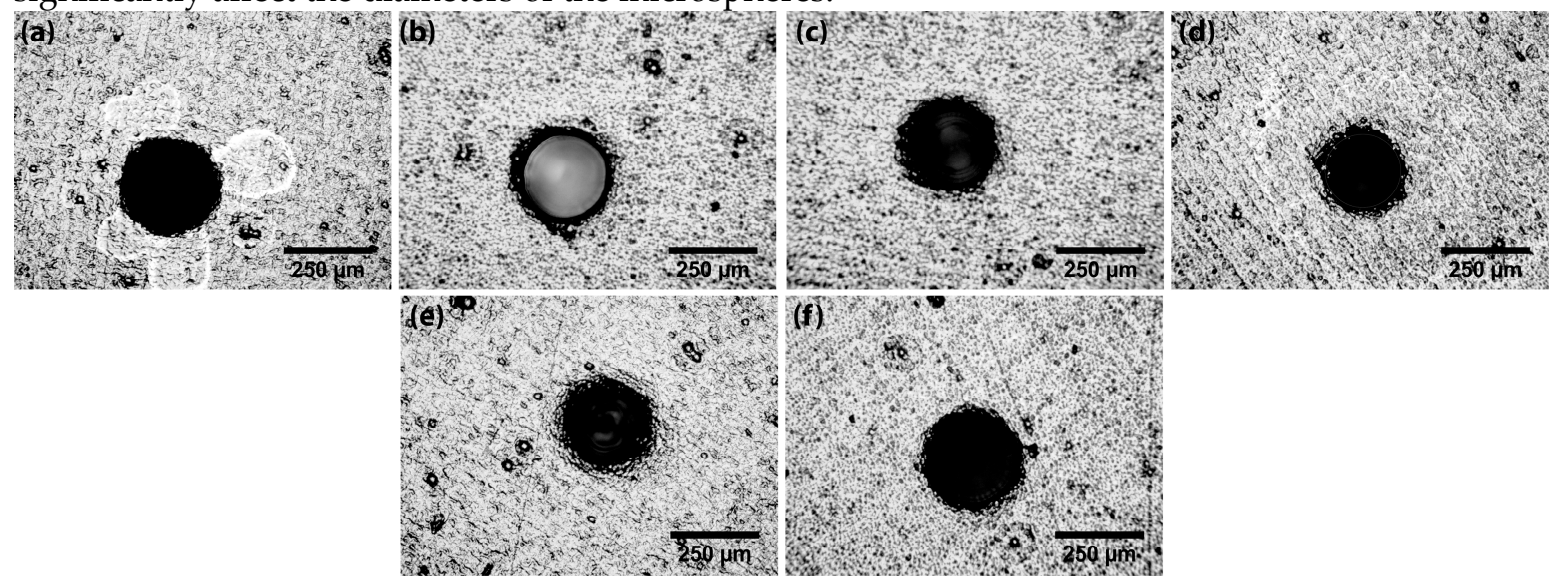

Figure 2. Characterization of the orifice in the device. Bright field images of the six orifices in the device.

Table 1. Diameters of orifices/nozzles in micrometers

\begin{tabular}{cc}
\hline Hole number & Diameter \\
\hline a & 246 \\
b & 235 \\
c & 216 \\
d & 210 \\
e & 222 \\
f & 246 \\
\hline
\end{tabular}

\subsection{Microsphere generation and crosslinking}

Respectively, a six-channel syringe pump (75900-50 from Cole Parmer) and PHD 2000 syringe pump (Harvard Apparatus, Holliston, MA) were used to continuously pump the PEGDA solution and mineral oil into the nozzle device. Tygon tubing (3350 - Fisher Scientific) was used to connect the device to the syringe pumps. A clamp holder fixed the device in a vertical position. This enhanced 
143

144

145

146

147

148

149

150

151

152

153

154

155

156

157

158

159

160

161

162

163

164

microsphere generation through gravity as the inertial force because the momentum of the fluid gave rise to a dynamic pressure in the channel. The microspheres produced by the device were crosslinked using an OmniCure S Series UV light system with an intensity of $40 \mathrm{~mW} / \mathrm{cm} 2$ for 30 seconds of exposure time at the outlet of the device and collected in DI water after the cross-linking process. The exposure was controlled by exposing only a certain portion of the tubing inside the UV box, which blocked the UV radiation from escaping. The microspheres were then centrifuged at 3,500 rpm for $10 \mathrm{~min}$ to remove the supernatant and washed in ethanol three times to remove the excess oil on the surfaces of the spheres. Successfully cross-linked PEGDA microspheres were then transferred to a 1xPBS solution and centrifuged three times to remove the ethanol for further analysis

\section{Results and discussion}

\subsection{Bright field imaging}

A Nikon Eclipse Ti microscope with a Zyla 4.2 camera (Andor) was used for the bright field imaging of the microsphere with $4 x$ and 10x objective lenses. NIS-Elements Advanced Research 4.30.02 software (Nikon) was used to capture the images of the PEGDA microspheres, as shown in Figure 3(a), (b), and (c). The flow-rate ratio is the ratio of the outer to the inner flows. Image software was used to measure the diameters of more than 1,000 microspheres for each condition, from the collected images. The experimental size distribution of the microspheres for the flow rate ratios of 180, 72, and 40 and the corresponding bright field images are shown in Figure 3(d), (e), and (f). The bright field images confirmed the uniformity of the microspheres and the size distributions had a consistent Gaussian profile. The average diameter of the microspheres for the highest flow rate ratio of 180 was $121 \pm 27 \mu \mathrm{m}$. The average diameter of the microspheres for the flow rate ratios of 72 and 40 were $196 \pm 63 \mu \mathrm{m}$ and $391 \pm 83 \mu \mathrm{m}$, respectively.

(a)

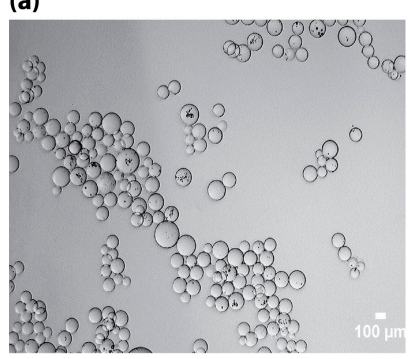

(b)

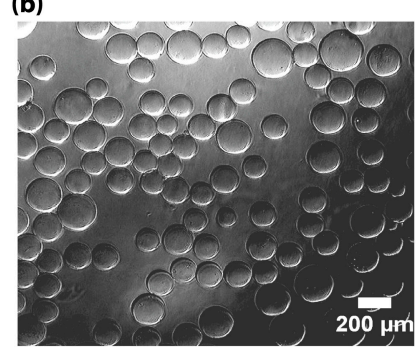

(c)

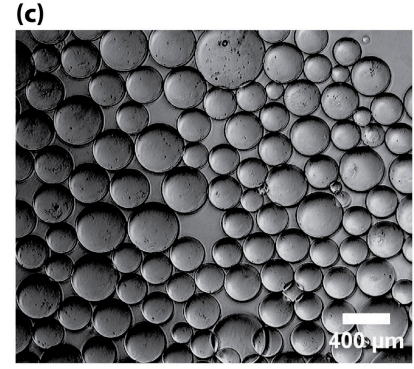

(d)

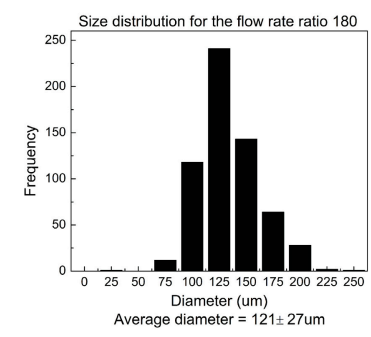

(e)

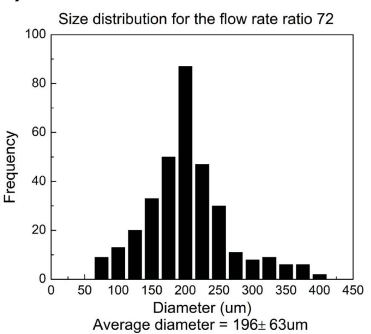

(f)

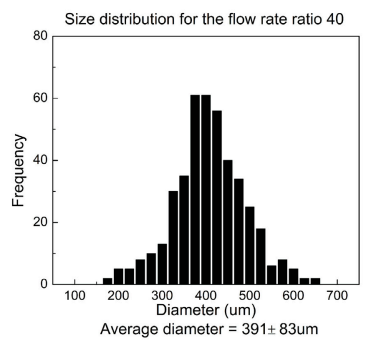

Figure 3. (a), (b), and (c) show the bright field images and (d), (e), and (f), show the size distribution characterization of the microspheres generated using the 3D coaxial multi-nozzle device for flow rate ratios of 180,72 , and 40. 
The average diameter of the PEGDA microsphere as a function of the flow rate ratio is plotted in Figure 4. As the flow rate ratio increased, the shear force at the nozzle increased to break the continuous media liquid. Hence, the average diameter of the microspheres was reduced from 1,200 $\mu \mathrm{m}$ to $121 \mu \mathrm{m}$ as the flow rate ratio increased from 2 to 180 . The theoretical microsphere diameter was obtained from Eq. 1 (with $\mathrm{D}=250 \mu \mathrm{m}$ and the proportionality constant $=2$ ), which agreed well with the experimental results shown in Figure 4. The smallest diameter of the microspheres was 121 $\pm 27 \mu \mathrm{m}$ since the nozzle diameter was limited due to the resolution of the 3D printer and the flow rates were limited by the pump capacities. A high standard deviation (SD) at a low flow rate ratio was observed because the shear force from the outer nozzle liquid was not high enough to consistently break the continuous medium flow into individual microspheres.

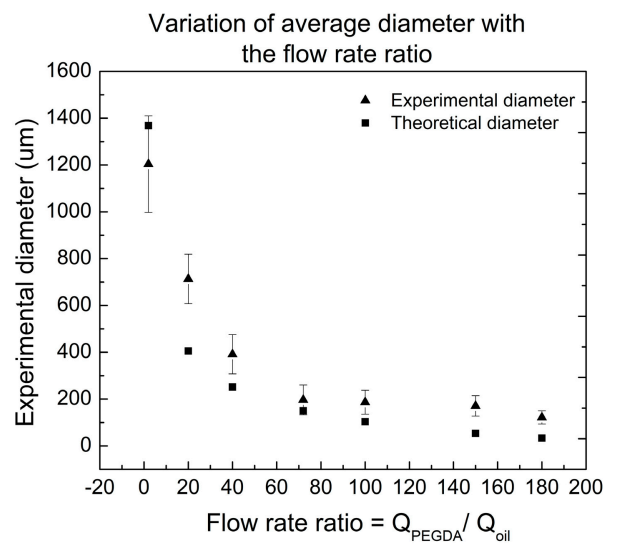

Figure 4. Variations in the experimental and theoretical microsphere diameters with the change in flow rate ratio. The theoretical diameter was obtained using Eq. 1. The flow rate ratio varied between 2 and 180. Error bars correspond to the standard deviations at the corresponding flow rates.

\subsection{High production rate}

A graph of the microsphere production rate as a function of the flow rate ratio between the meniscus and continuous media is shown in Figure. 5. As the average diameter of the microsphere decreased, the number of spheres produced per unit volume of inner liquid increased. Hence, as the flow rate ratio increased from 2 to 180, the production rate of the microsphere increased from 200 microspheres per sec to as high as $2.12 \mathrm{e}+5$ microspheres per sec $(0.25 \mathrm{~mL} / \mathrm{min})$. In this device design, the shear force from the outer liquid is applied from $360^{\circ}$ radial directions on the inner liquid contrary, while its planar counterparts have only a two-dimensional shear force. This enables the generation of microspheres with smaller dimensions at higher production rates for the same flow rates. To the best of our knowledge, this production rate per nozzle is four times higher than the highest production rate PDMS devices found in the literature [23], and the highest production rate demonstrated among devices with multiple nozzles fabricated using stereolithography [28, 34-36, 42, 45].

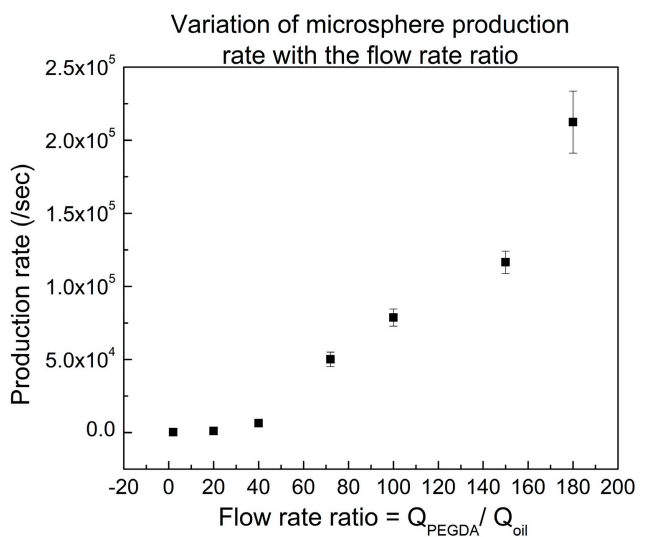


Figure 5. Scatter plot of the production rate as a function of the flow rate ratio. The flow rate ratio of the PEGDA solution varied between 2 and 180.

\section{Conclusions}

We have successfully developed a multi-nozzle coaxial flow-focusing device capable of generating PEGDA microspheres and demonstrating a high microsphere production rate of 2.2e+5 microspheres/sec. This device has four times higher a production rate per nozzle than others published recently. This is a mask-less, low cost, clean room free method for making high production rate microfluidic microsphere production devices. The generated microspheres are able to offer a localized and prolonged delivery of genes, protein, or stem cells. Such cells could serve as harbor cells in 3D tissue regeneration scaffolds that can be used to treat diseases such as osteoarthritis.

Funding: This research is supported by NSF CBET 1264848 and NIH RO1-B214765.

\section{References}

1. "Tissue engineering," Nature Biotechnology 18, IT56 (2000).

2. K. Widder, G. Flouret, and A. Senyei, Journal of Pharmaceutical Sciences 68.1, 79 (1979).

3. S. Hong, H. Hsu, R. Kaunas, and J. Kameoka, Lab Chip 12, 3277 (2012).

4. P. Garstecki, I. Gitlin, W. DiLuzio, G. Whitesides, E. Kumacheva, and H. Stone, Appl. Phys. Lett. 85, 2649 (2004).

5. T. Nisisako, T. Torii, and T. Higuchi, Chemical Engineering Journal 101, 23 (2004).

6. T. Zhang, X. Zhang, X. Yan, L. Kong, G. Zhang, H. Liu, J. Qiu, and K. Yeung, Chemical Engineering Journal 228, 398 (2013).

7. Y. Lan, L. Yang, M. Zhang, W. Zhang, and S. Wang, Applied Materials and Interfaces 2.1, 127 (2010).

8. L. Yang, M. Zhang, Y. Lan, and W. Zhang, New J. Chem. 34, 1355 (2010).

9. R. Lanza, W. Kühtreiber, D. Ecker, J. Staruk, and W. Chick, Transplantation 59.10, 1377 (1995).

10. J. Champion, Y. Katare, and S. Mitragotri, Journal of Controlled Release 121, 3 (2007).

11. X. Liu, X. Jin, and P. Ma, Nature Materials 10 (2011).

12. T. Ward, M. Faivre, M. Abkarian, and H. Stone, Electrophoresis 26, 3716 (2005).

13. Q. Xu, M. Hashimoto, T. Dang, T. Hoare, D. Kohane, G. Whitesides, R. Langer, and D. Anderson, Small $5.13,1575$ (2009).

14. A. Jaklenec, E. Wan, M. Murray, and E. Mathiowitz, Biomaterials 29.2, 185 (2008).

15. Q. Qiu, P. Ducheyne, and P. Ayyaswamy, Journal of Biomedical Materials Research 52.1, 66 (2000).

16. M. Seo, C. Paquet, Z. Nie, S. Xu, and E Kumacheva, Soft Matter 3, 986 (2007).

17. W. Li, E. Young, M. Seo, Z. Nie, P. Garstecki, C. Simmons, and E. Kumacheva, Soft Matter 4, 258 (2008).

18. N. Wu, Y. Zhu, S. Brown, J. Oakeshott, T. Peat, R. Surjadi, C. Easton, P. Leech, and B. Sexton, Lab Chip 9, 3391 (2009).

19. D. Conchouso, D. Castro, S. Khan, and I. G. Foulds, Lab Chip 14, 3011 (2014).

20. H. Wu, P. Lei, G. Liu, Y. Shrike-Zhang, J. Yang, L. Zhang, J. Xie, W. Niu, H. Liu, J. Ruan, Y. Hu, and C. Zhang, Scientific Reports 7.1, 2045 (2017).

21. Y. Pang, H. Kim, Z. Liu, and H. Stone, Lab Chip 14, 4029 (2014).

22. E. Amstad, M. Chemama, M. Eggersdorfer, L. Arriaga, M. Brenner, and D. Weitz, Lab Chip 16, 4163 (2016).

23. H. Jeong, V. Yelleswarapu, S. Yadavali, D. Issadore, and D. Lee, Lab Chip 15, 4387 (2015).

24. H. Jeong, D. Issadore, and D. Lee, Korean J. Chem. Eng. 33.6, 1757 (2016).

25. T. Nisisako and T. Torii, Lab Chip 8, 287 (2008).

26. T. Nisisako, T. Ando, and T. Hatsuzawa, Lab Chip 12, 3426 (2012).

27. M. Muluneh and D. Issadore, Lab Chip 13, 4750 (2013).

28. D. Conchouso, D. Castro, S. Khan, and I. Foulds, Lab Chip 14, 3011 (2014).

29. I. Kobayashi, Y. Wada, K. Uemura, and M. Nakajima, Microfluid Nanofluid 8, 255 (2010).

30. M. Kendall, D. Bardin, R. Shih, P. Dayton, and A. Lee, Bubble Science, Engineering \& Technology 4.1 (2012).

31. G. Tetradis-Meris, D. Rossetti, C. de Torres, R. Cao, G. Lian, and R. Janes, Ind. Eng. Chem. Res. 48, 8881 (2009).

32. S. Yoon, Z. Horita, H. Kim, and J. Mater. Process. Tech. 201, 32 (2008).

33. S. Yoon, H. Jeong, S. Lee, and H. Kim, Comp. Mater. Sci. 77, 202 (2013). 
34. Y. Morimoto, W. Tan, and Shoji Takeuchi, Biomed Microdevices 11, 369 (2009).

35. Y. Morimoto, K. Kuribayashi-Shigetomi, and S. Takeuchi, J. Micromech. Microeng 21, 54031 (2011).

36. Y. Tsuda, H. Onoe, and S. Takeuchi, in 14th International Conference on Miniaturized Systems for Chemistry and Life Sciences, Groningen, The Netherlands, 3-7 October 2010.

37. R. Parenteau-Bareil, R. Gauvin, and F. Berthod, Materials 3, 1863 (2010).

38. R. Shah, H. Shum, A. Rowat, D. Lee, J. Agresti, A. Utada, L. Chu, J. Kim, A. Fernandez-Nieves, C. Martinez, and D. Weitz, Mater. Today 11, 18 (2008).

39. S. Huang, W. Tan, F. Tseng, and S. Takeuchi, Micromech. Microeng. 16, 2336 (2006)

40. T. Tran, S. Cater, and A. Abate, Biomicrofluidics 8, 016502 (2014).

41. P. Garstecki, A. Ganan-Calvo, and G. Whitesides, Technical Sciences 53.4 (2005).

42. S. Jaligama, P. Huang, and J. Kameoka, in 2016 38th Annual International Conference of the IEEE Engineering in Medicine and Biology Society (EMBC), Orlando, FL, 2016, pp. 4220-4223.

43. J. Vogel and E. Boschetti, U.S. patent 7,338,657 B2 (4 March 2008).

44. R, Mehrotra and J. Kameoka, Applied Physics Letter 92, 213109 (2008).

45. T. Femmer, A. Jans, R. Eswein, N. Anwar, M. Moeller, M. Wessling, and A. J. Kuehne, ACS Appl. Mater. Interfaces 7, 12635 (2015). 